\title{
Observation of nanosecond laser induced fluorescence of in vitro seawater phytoplankton
}

\author{
Thomas J. Bensky, ${ }^{1, \star}$ Lisa Clemo, ${ }^{1}$ Chris Gilbert, ${ }^{2}$ Bryan Neff, ${ }^{1}$ \\ Mark A. Moline, ${ }^{3}$ and Dov Rohan ${ }^{1}$ \\ 1Department of Physics, California Polytechnic State University, San Luis Obispo, California 93407, USA \\ ${ }^{2}$ Department of Electrical Engineering, California Polytechnic State University, San Luis Obispo, California 93407, USA \\ ${ }^{3}$ Department of Biological Sciences, California Polytechnic State University, San Luis Obispo, California 93407, USA
}

\begin{abstract}
Seawater has been irradiated using a train of $70 \mathrm{~ns}$ flashes from a $440 \mathrm{~nm}$ laser source. This wavelength is on resonance with the blue absorption peak of Chlorophyll pigment associated with the photosystem of in vitro phytoplankton. The resulting fluorescence at $685 \mathrm{~nm}$ is instantaneously recorded during each laser pulse using a streak camera. Delayed fluorescence is observed, yielding clues about initiation of the photosynthetic process on a nanosecond time scale. Further data processing allows for determination of the functional absorption cross section, found to be $0.0095 \AA^{2}$, which is the first reporting of this number for in vitro phytoplankton. Unlike other flash-pump studies of Chlorophyll, using a LED or flashlampbased sources, the short laser pulse used here does not reveal any pulse-to-pulse hysteresis (i.e., variable fluorescence), indicating that the laser pulses used here are not able to drive the photosynthetic process to completion. This is attributed to competition from a back reaction between the photoexcited photosystem II and the intermediate electron acceptor. The significance of this work as a new type of deployable ocean fluorimeter is discussed, and it is believed the apparatus will have applications in thin-layer phytoplankton research.
\end{abstract}

\section{Introduction}

We report on the observation of fluorescent emission of in vitro seawater phytoplankton as a response to a train of $440 \mathrm{~nm}$ laser flashes, each lasting approximately $70 \mathrm{~ns}$, arriving at $1 \mathrm{~ms}$ intervals. Choosing to work on fluorescence in the nanosecond regime was motivated by a larger effort to develop an inexpensive, laser-based, deployable ocean-plankton fluorimeter (discussed below), using as many off-the-shelf optical components as possible. The success of a LED or flashlamp-based fluorimeters is acknowledged $[1,2]$, but a laser-based fluorimeter has two primary strengths that this effort seeks to exploit. First, optical harmonics of diode pumped lasers now make an off-the-shelf $440 \mathrm{~nm}$ laser readily available, maximizing efficiency of Chlorophyll-a (Chl-a) pumping. Second, since the laser is naturally collimated, fluorescence can be stimulated along the entire volume of water traversed by the laser beam, lifting spatial restrictions of non-laser-based fluorimeters. It is hoped this will open up the possibility of performing real-time spatial mapping of plankton communities, via their fluorescence, over a distance of several meters from the pump source.

Prior work in this area has revealed that the photosynthetic apparatus (PA) is marvelously diverse in its response to optical stimulation. Pumping with picosecond light sources will reveal similarly 
timed fluorescent events, as will pumping with nanosecond, microsecond, and millisecond flashes. Each regime has its value in revealing the nature of energy migration through the PA, and it appears as if light with longer pump durations is able to probe deeper into the long succession of steps that ultimately leads to stable charge separation and stored energy [3]. Picosecond pump sources, for example, do not probe the PA at all, reporting only the singlet lifetime of (Chlorophyll) Chl antenna [4-6], perhaps even before any energy is transferred to the PA. Nanosecond pump sources, used in the work presented here, do reveal initial traversal of the pump energy from the Chl-a as "light antenna" into the PA, and allow for monitoring the early attempts of the PA at charge separation $[7,8]$. The photosynthetic reactions induced with these short pump pulses, however, are unstable and fluorescence results from their reversal, as seen in Ref. [7], and in this paper. Microsecond pump sources probe so deeply as to even allow for measurement of oxygen evolution in the PA [9]. Finally, millisecond pump sources are perhaps the most prolific and probe deeply into the dynamics of the PA, even resolving closure of the PA to additional photons as photosynthesis and charge separation occur [3,10-13].

\section{Instruments and Methods}

Specific details concerning the construction of the apparatus used in this work will be presented in a forthcoming publication [14]. Briefly, a $Q$-switched, $1 \mathrm{kHz}$, diode pumped, Nd:YLF laser [15] is used to stimulate the Chl-a fluorescence. The laser has a spectral peak at $440 \mathrm{~nm}$ with a bandwidth of approximately $20 \mathrm{~nm}$ at half-maximum. The temporal laser pulse width is also Gaussian shaped, with a full width at half-maximum of approximately $50 \mathrm{~ns}$. Time averaged output power is $10 \mathrm{~mW}$, corresponding to a peak pulse power of $200 \mathrm{~W}$ and a pulse intensity of $6.4 \times 10^{7} \mathrm{~W} / \mathrm{m}^{2}$, or $233 \mathrm{~mol}$ quanta $\mathrm{s}^{-1} \mathrm{~m}^{-2}$. The streak camera is a bare 20 Model ST-X streak tube [16] that is powered with circuits constructed inhouse. The resulting streak image is captured using a thermoelectrically cooled, integrating CCD camera. Synchronization between the sweep voltage of the streak camera and the laser/sample traversal is ensured using a precision delay generator [17]. The seawater samples used in this work are live samples of ocean water collected near a university-owned pier facility at Avila Beach, California, which extends about $1 \mathrm{~km}$ into San Luis Obispo Bay, located on the central California coast at $35.3 \mathrm{~N}, 120.6 \mathrm{~W}$. A sample was transported to the laboratory and placed in the laser beam within 15 min after collection. Enough seawater is collected to fill a $30 \mathrm{~cm}$ water column. Scattering of the blue laser beam by particulates in the seawater clearly reveals the thin blue laser beam by direct observation. The same observation through a $692( \pm 40) \mathrm{nm}$ (red) filter (Edmund Optics 48148) reveals a faint red glow emanating from the same volume traversed by the laser beam. In normal operation, two filters are placed in front of the streak camera aperture, the 692 bandpass filter, and a $440 \mathrm{~nm}$ band-reject filter, which blocks scattered laser light from entering the streak camera. Data presented in this paper represent typical observations from eight samples collected between January 2006 and March 2007. A schematic for the assembled apparatus is shown in Fig. 1. The water column, laser beam, and streak camera aperture are mutually parallel allowing an image of the thin volume of water traversed by the laser beam to be captured by the streak camera.

\section{Results}

A typical streak image taken for live seawater is shown in Fig. 2. As shown, the vertical extent of the streak image represents approximately $30 \mathrm{~cm}$ of a laboratory water column; the entire horizontal extent of the frame represents $150 \mathrm{~ns}$ of time. The laser enters the water column at the bottom of the image, at a time of $0 \mathrm{~ns}$. The bright features near 10 and $30 \mathrm{~cm}$ are surface features due to water-glass and water-air interactions from the container holding the seawater itself and are ignored in favor of a surface free region near the center of the water column, near $20 \mathrm{~cm}$. A single image from the streak camera is considered as a collection of pixels in a (time, distance) coordinate space. Each vertical pixel represents a discrete volume of seawater a given distance from the bottom of the container, while each horizontal pixel represents the time evolution of the fluorescence of a given volume after irradiation. The sweep voltage for the streak camera we used makes each horizontal pixel represent an additional $1 \mathrm{~ns}$ in time evolution.

For analysis, a row of pixels was chosen at the middle of the image in Fig. 2, near $20 \mathrm{~cm}$. Using image analysis software [18], a horizontal pixel intensity profile is extracted from the image and plotted as the fluorescence curve in Fig. 3. The horizontal axis is the same time axis as in the raw streak image. The right vertical axis, in its rawest form, would be a digital pixel value from 0 to 4098 (from the 12 bit

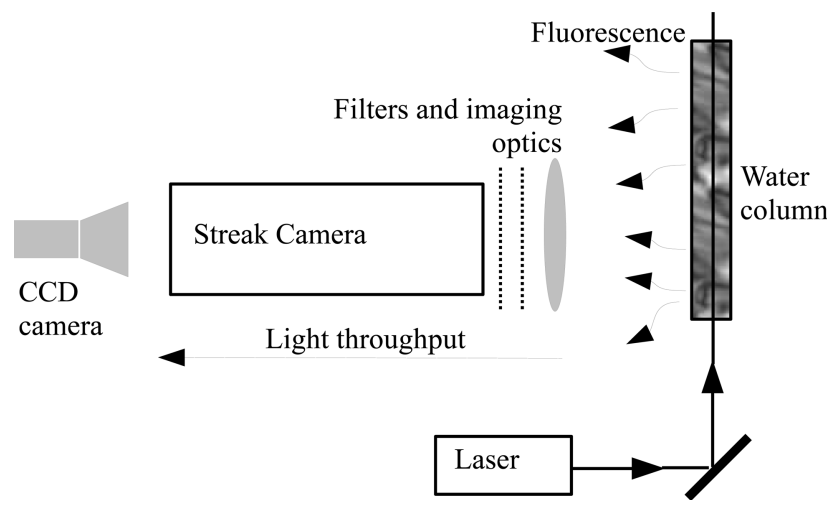

Fig. 1. Schematic of the apparatus used in this work. The laser pumps a column of seawater, oriented parallel to the slit of a streak camera. Imaging optics creates an image of the water traversed by the laser beam onto the aperture of the streak camera, after passing through a blue-reject and $685 \mathrm{~nm}$ bandpass filter. A CCD camera is used to capture the resulting streak camera output 


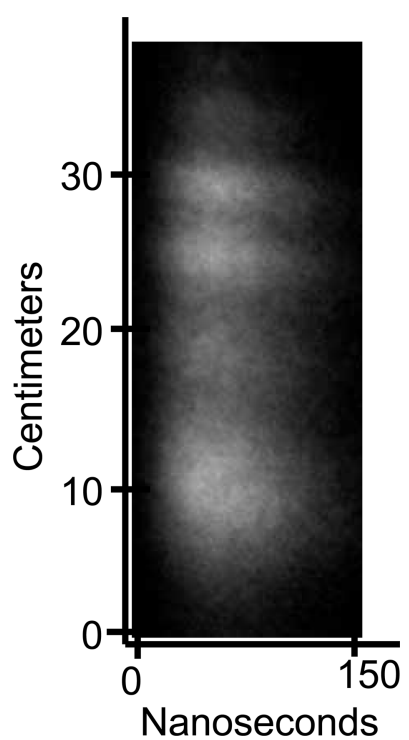

Fig. 2. Typical streak image obtained for in vitro seawater.

CCD camera) sampled along the horizontal line in the image in Fig. 2 at $20 \mathrm{~cm}$. For this paper, however, these pixel values were calibrated by tabulating pixel intensity values observed as a result of sending laser pulses with known powers [19] into the streak camera at the same wavelength as the fluorescence. Hence the right vertical axis in Fig. 3 is fully calibrated in microwatts. The vertical axis of the streak camera represents the spatial extent of a sample traversed by the laser beam. However, because of the surface features discussed above, any extended use of this axis is ignored. Thus attenuation coefficients are not presented in this paper, but will be an important analysis point in the ocean-deployable version of this apparatus.

To make correlations with the pump light, another streak image was acquired with the laser reject filter removed. In this configuration, the $440 \mathrm{~nm}$ scattered laser light dominates the fluorescence light, and the streak camera effectively captures the time evolution of the laser pulse. A pixel intensity profile is taken for this second streak image (at the same vertical position as the first) and is plotted in Fig. 3 as the laser curve, with a calibrated power axis on the left. When viewing Fig. 3, the reader is cautioned to observe the vertical axes carefully. The laser intensity values are along the left vertical axis, while the fluorescence intensity values are along the right vertical axis. There is a factor of $10^{8}$ between the two scales. This figure essentially represents the raw data this apparatus is able to capture: instantaneous fluorescent emission from a seawater sample during the evolution of the excitation (or pump) light pulse.

\section{Discussion of Data}

\section{A. Temporal Features}

In examining Fig. 3, it is noted that the horizontal (time) axis is absolute. The point "time $=0$ " represents when the laser is preparing to fire, starting a measurement cycle. Light from the laser pulse is seen first, followed by the fluorescent emission from $\mathrm{Chl}$, presumably Chl-a. The most prominent temporal feature is an approximate $10 \mathrm{~ns}$ delay between the pump and the fluorescent light emission curves. This delay was consistently observed over several months, with eight different seawater samples, and varied by $( \pm 2) \mathrm{ns}$. It is tempting to call the delay instrumental, but this is difficult to justify given that the only experimental difference between the two curves in Fig. 3 is the removal of the laser light rejection filter. Light travel times cannot be the cause, since a $10 \mathrm{~ns}$ delay would represent some spatial gap of approximately $3 \mathrm{~m}$, which is two to three times larger than the entire apparatus. Such a delay between pump and fluorescent emission has been seen in other work [20] and is common in photosynthetic systems [7,21-27], being called delayed fluorescence (DF). It is noted that, with the exception of Ref. [20], these references do not directly show evidence of $\mathrm{DF}$, as shown in Fig. 3 here. This is attributed to the fact that, to our knowledge, this work is the first to observe fluorescence of seawater during a laser flash, with a detector having such an extremely large temporal bandwidth at correspondingly short time scales. Two elements of control against the live seawater samples have been performed.

The first control is a set of data acquired using a sample consisting of fresh spinach leaves soaked in acetone for approximately $2 \mathrm{~h}$. The sample was filtered of any visible particulates then placed in the laser beam. The sample is seen to fluoresce very brightly in the red. Apart from a multiplicative constant in amplitude, the temporal shapes of the laser pulse and spinach fluorescence responses are identical; the time delay observed in Fig. 3 is absent. The absence of a delay in the spinach sample makes physical sense, as follows. The Chl-a in the acetone solution is not connected to a photosynthetic apparatus; it is a dead sample. In such a solution, the Chl-a molecules absorb laser photons and subsequently

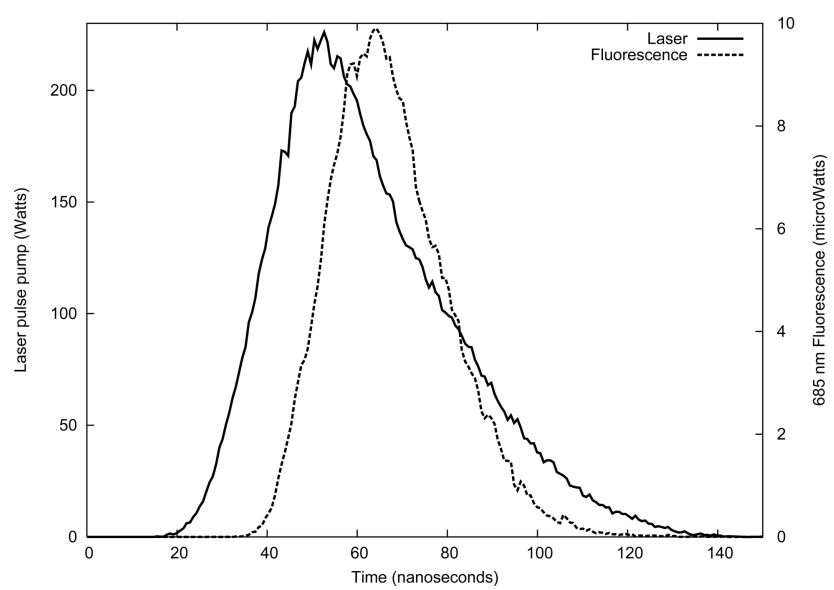

Fig. 3. Laser (solid curve) versus fluorescence (dotted curve) as extracted from a horizontal row near $20 \mathrm{~cm}$ in Fig. 2. The laser curve was taken independently from the fluorescence curve, with the laser-light reject filter removed. 
fluoresce with a lifetime lasting approximately $2 \mathrm{~ns}$ (see picosecond references above), which is too fast to observe during the $70 \mathrm{~ns}$ laser pulse we used. During the laser pulse, therefore, there is a continual pump-fluoresce-pump-fluoresce cycle occurring. The short fluorescent lifetime, relative to the laser pulse width, means that, at any given instant, there is a supply of unexcited or recently deexcited Chl-a electron transitions that can (re)absorb a pump photon and subsequently fluoresce. More pump photons yields more fluorescence and the overall fluorescence response simply follows the temporal envelope of the laser pulse itself. (As a small additional control step sample, off-the-shelf distilled water was placed in the apparatus, which yielded no detectable signal.)

The second control was in additional measurements on the seawater signal as follows. Upon careful inspection of Fig. 3, it is evident that, although the rising edge of both the laser and the fluorescence pulses are similar in appearance, the laser pulse clearly has a longer decaying "tail," making it appear to last approximately $9 \mathrm{~ns}$ longer than the fluorescence pulse. This leads to the possibility that the fluorescence signal is actually stimulated Raman scattering. This possibility has been eliminated with two tests. The first test is in a light spectrum collected from the volume of seawater traversed by the laser beam, taken with an Ocean Optics USB 4000 spectrometer [28]. The spectrometer returns intensity versus wavelength of an injected light sample in real time. For the seawater sample, a strong peak at $440 \mathrm{~nm}$ was observed, in addition to an $\approx 20 \mathrm{~nm}$ wide feature centered near $690 \mathrm{~nm}$. The overall spectrum closely resembles the "Chlorophyll-a" curve found on page 55 of Ref. [12]. In other words, to within the detectability of the spectrometer, the spectrum looks very "clean," unlike that expected from light dominated by stimulated Raman scattering, which would contain many more features spread throughout the spectral region of interest. The second test is in the observation of the temporal laser-pulse profile generated by the streak tube, as a function of laserlight power scattered into the tube. Observations reveal an overall temporal intensity-broadening of the laser pulse, by the phosphor screen, at full laser power. This is attributed to the phosphor being inundated with higher kinetic energy electrons, as generated by the higher frequency laser photons. Upon impact, the phosphor glows very strongly, which "bleeds" into neighboring pixels on the phosphor. As the laser pulse is attenuated using a variable neutral density filter, it is observed to decrease in width, relative to its fixed peak. In particular, the long tail seen from 80 to $140 \mathrm{~ns}$ in Fig. 3 disappears, causing the laser pulse and fluorescence to share a nearly identical temporal width. Since the peaks in the laser and fluorescence curves do not shift as a function of input laser power, Fig. 3 is presented to illustrate the maximum fluorescence signal attainable for seawater, given all available laser pump power produced by the off-the-shelf laser we used.
The lack of a delay in fluorescence in the acetone sample indicates the DF observed in the seawater (or "live") sample comes from some connection between the Chl-a antenna and the photosynthetic apparatus (see below). Despite this connection, however, the live data still do not reveal any pulse-topulse dynamics. That is, the fluorescence shown in Fig. 3, due to laser pulse $N$ is not affected by laser pulse $N-1$, and will not affect the fluorescence caused by laser pulse $N+1$. This indicates a disconnection between the Chl-a antenna and the photosynthetic apparatus. This apparent paradox sets a limit for what the pump source that we used is able to achieve relative to the photosynthetic process: the DF indicates that the photosynthetic process has started in the live sample, but the lack of pulse-topulse dynamics indicates that the process then fails to sustain itself over the $1 \mu \mathrm{s}$ off time between laser pulses. It is concluded then that stable charge separation is not achieved with a pump source lasting a few tens of nanoseconds, and any initiated photosynthetic processes identifiable via fluorescence terminates with each laser pulse. This is in stark contradistinction with studies performed with a LED or flashlampbased pump sources, where the pump durations last up to several orders of magnitude longer in time $[1,2]$. It appears that pumping a live sample in the nanosecond regime is at an awkward boundary in the photoinitiation of photosynthesis. Such pulses are too long to observe any fluorescent lifetimes, and too short to fully initiate the photosynthetic process.

Evidence of the connection between the $10 \mathrm{~ns}$ DF observed in this work and the PA is as follows. Figure 4 shows an adaptation of the early stages of the Z-Scheme for photosynthesis from Refs. [21,22]. In this scheme a $440 \mathrm{~nm}$ photon is absorbed by a Chl-a antenna. After nonradiative photon energy losses, an electron is promoted from state (0) to (1), creating $\mathrm{Chl}^{*}$, an excited $\mathrm{Chl}$ molecule. As indicated in Fig. 4, within $60 \mathrm{ps}, \mathrm{Chl}^{*}$ transfers its energy to P680, the photosystem II reaction center forming $P^{*}\left(\right.$ or $\left.P 680^{*}\right)$. Within $3 \mathrm{ps}$, the first step in charge separation can occur, where I (pheophytin) accepts the excited electron from the photosystem, producing oxidized $P^{+}$and reduced $I^{-}$states. Finally, $P^{+} I^{-} Q_{A}$ can revert to $P^{*}$ for prompt electron deexcitation via fluorescence at $685 \mathrm{~nm}$ within a lifetime that seems somewhat undetermined, but consistent with

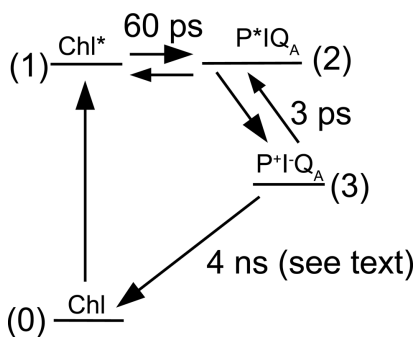

Fig. 4. Initial steps in the "Z-diagram" of photosynthesis adapted from Ref. [22]. It is believed the $70 \mathrm{~ns}$ laser pulse we used cannot compete with the $4 \mathrm{~ns}$ recombination step between $P^{+}$and $I^{-}$. 
the $8-12$ ns DF observed here. Reference [22] puts this time at $4 \mathrm{~ns}$, Ref. [7] puts this time between 15 and $60 \mathrm{~ns}$, and Ref. [21] reports between 100 and $300 \mathrm{~ns}$. It is also noted that all these studies use different samples in different environments. The lifetime of the $P^{+}$and $I^{-}$recombination back reaction occurs within the time of the DF observed in this work, prompting the belief that this back reaction is responsible for the DF observed. Any further charge separation, for example, to $Q A^{-}$, the secondary plastoquinone electron acceptor, does not occur, since this step involves dynamic processes lasting milliseconds to seconds, which are simply not seen in this work. It is concluded then that a $70 \mathrm{~ns}$ laser pulse cannot drive the photosynthetic process past the reduction of I (the pheophytin).

It is noted that fluorescence at the red wavelength $(685 \mathrm{~nm})$ can only come from deexcitation of the $\mathrm{Chl}$ antenna. Thus, a $10 \mathrm{~ns}$ delay in fluorescence means an excited electron must be prevented from deexcitation for this time duration. This cannot be explained by deexcitation of the Chl-a antenna alone, since this fluoresce is prompt, with a $2 \mathrm{~ns}$ lifetime. If, however, charge separation is initiated via the pheophytin electron acceptor, a delay in fluorescence is possible as the back reaction takes its respective time to complete, while the excited electron is energetically separated from the Chl antenna and not available for deexcitation and fluorescence.

\section{B. Fluorescent Yield}

The data shown in Fig. 3 allow for a direct determination of the fluorescent yield from the Chl-a as a function of instantaneous pump intensity. This result is shown in Fig. 5, where the ratio of fluorescent emission to laser fluence is computed at a given time during the laser pulse. This result allows for comparison of this paper with that of others [12,29]. In particular, as shown in Ref. [30], fluorescent yield follows the "one hit Poisson" function $1-e^{-\sigma J}$, where $J$ is the laser fluence and $\sigma$ is the functional cross section. For the relatively small number of photons in a single laser pulse (a factor of 1000 lower than that used in Fig. 2 of Ref. [14]), $\sigma J \ll 1$, so $e^{-\sigma J}=1-\sigma J$, meaning the Poisson function reduces to simply $\sigma J$. Hence, the slope of the data shown in Fig. 5 is the functional cross section, found to be $9.46 \times 10^{-19} \mathrm{~cm}^{2}$ or 0.0095 $( \pm 0.0002) \AA^{2}$.

This cross section for in vitro seawater has not been found in the literature, and this may be the first reporting of it. The closest variant, as derived from fluorescence yield measurements, has been reported for Chlorella Vulgaris at 1.3 to $280 \AA^{2}$ [31,32]. Another report shows this number to be dependent on ambient lighting conditions and flash energy [30] with values as low as $0.29 \AA^{2}$, which is (merely) a factor of 30 larger than the value found here for seawater. It is not possible to decide on the correctness of this cross section for seawater; instead it is pleasing that a cross section, with a somewhat similar value to the work of others, can be

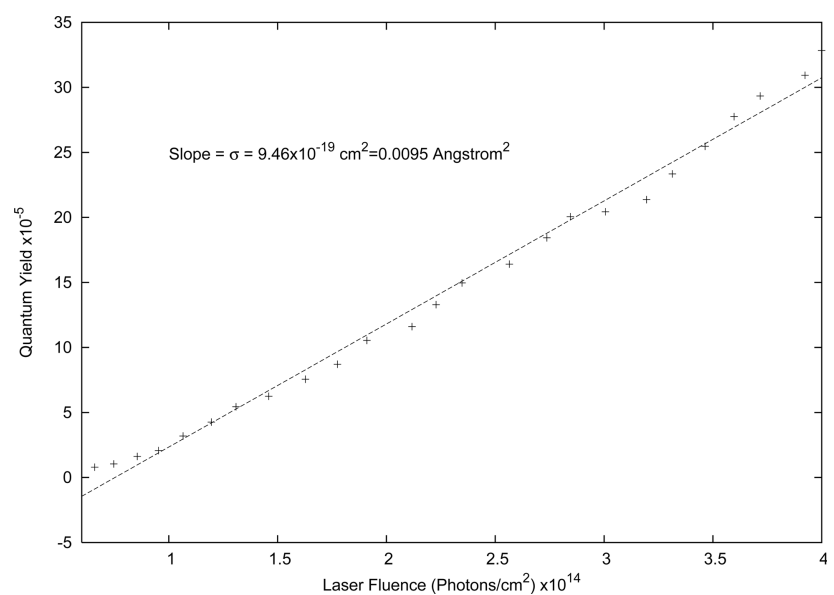

Fig. 5. Fluorescent yield versus laser fluence, as derived from the data shown in Fig. 3 by eliminating time between the two curves. The slope is the functional cross section for in vitro seawater phytoplankton.

derived here, considering the extremely different circumstances under which it was taken. Further work is needed to quantify the place of this result.

\section{Use as a Deployable Ocean Fluorimeter}

As mentioned in Section 1, this work is part of a larger effort to develop a deployable, laser-based, ocean fluorimeter. The two parameters discussed above, the time lag of DF, and the functional cross section came by analyzing pixel intensities across only a single horizontal axis of an acquired streak image. Other horizontal rows, representing the spatial axis of the streak camera (due to laboratory constraints) went completely unused in this paper. It is hoped this spatial axis will prove useful in real-time phytoplankton mapping applications for the eventual ocean fluorimeter. In use, the DF can be observed and the cross section derived for every horizontal row of pixels acquired from a streak camera image, representing (with suitable optics) many vertical feet of instantaneous phytoplankton fluorescent response to each pulse of the laser.

Current work involves integrating this entire apparatus onto a REMUS [33] platform. Initial goals when deployed in the ocean will be to monitor the $\mathrm{DF}$ and functional cross section along the full spatial capability of the streak camera and attempt to attach the results to global oceanographic parameters.

\section{Conclusions}

En route to developing a laser-based ocean fluorimeter, we observed instantaneous fluorescent emission from $\mathrm{Chl}$ in phytoplankton resident in in vitro seawater samples as a result of pumping with a $440 \mathrm{~nm}, 70 \mathrm{~ns}$ laser pulse. Delayed fluorescence of $10( \pm 2) \mathrm{ns}$ is seen, and a functional cross section of $0.0095 \AA^{2}$ is derived from the data. The delayed fluorescence is connected to a back reaction in the early stages of the charge-transfer process of photosynthesis. The data do not reveal any pulse-to-pulse hysteresis, such as that seen with pump sources with 
flashes lasting milliseconds. The lack of pulse-topulse hysteresis may prove useful for direct phytoplankton mapping as a function of concentration since the fluorescent emission from the plankton will be linear with pump energy. This linearity is a desirable feature for an ocean fluorimeter, not possible with systems exhibiting variable fluorescence, since fluorescence emission in these regimes has a complicated, nonlinear dependence on pump energy and relative irradiation time.

Finally, future work includes deploying the apparatus into the open ocean and monitoring the delayed fluorescence and cross section as a function of location and depth [34]. It is hoped either or both of these parameters will yield clues to the instantaneous phytoplankton taxonomy and/or physiology relative to the local ocean environment. Finally, it is hoped the spatial discretization of seawater, made possible by the streak camera, will allow for increased monitoring resolution of thin-layer, coherent, phytoplankton patches [35].

The authors gratefully acknowledge funding from the National Science Foundation under grant OCE0428929. T. J. Bensky thanks M. Moelter of the California Poytechnic State University Physics Department for a careful proofreading of this manuscript. We also thank the anonymous reviewers for helping us to clarify various claims made in this paper.

\section{References}

1. Z. Kolber, O. Prasil, and P. Falkowski, "Measurements of variable chlorophyll fluorescence using fast repetition rate techniques: defining methodology and experimental protocols," Biochim. Biophys. Acta 1367, 888-106 (1988).

2. P. Falkowski and Z. Kolber, "Variations in chlorophyll fluorescence yields in phytoplankton in the world oceans," Aust. J. Plant Physiol. 22, 341-355 (1995).

3. G. Papageorgiou and Govindjee, Chlorophyll a Fluorescence: a Signature of Photosynthesis (Springer, 2004).

4. G. Portrer, J. A. Synowiec, and C. J. Tredwell, "Intensity effects on the fluorescence of in vivo chlorophyll," Biochim. Biophys. Acta 11, 329-3361 (1977).

5. J. Ide, D. Klug, W. Kuhlbrandt, L. Giorgi, and G. Porter, "The state of detergent solubilised light-harvesting chlorophyll-a/b protein complex as monitored by ps time-resolved fluorescence and circular dichroism," Biochim. Biophys. Acta 893, 349-364 (1987).

6. A. J. Campillo, S. L. Shapiro, V. H. Kollman, K. R. Winn, and R. C. Hyer, "Picosecond exciton annihilation in photosynthetic systems," Biophys. J. 16, 93-97 (1976).

7. M. Mimuro, S. Akimoto, T. Tomo, M. Yokono, H. Miyashita, and T. Tsuchiya, "Delayed fluorescence observed in the ns time region at $77 \mathrm{~K}$ orginated directly from the photosystem II reaction center," Biochim. Biophys. Acta Mol. Basis Dis. 1767, 327-334 (2007).

8. P. Booth and J. Paulsen, "Assembly of light-harvesting chlorophyll $\mathrm{a} / \mathrm{b}$ complex in vitro. Time-resolved fluorescence measurements," Biochemistry 25, 5103-5108 (1996).

9. V. Goltsev, T. Ortoidze, Z. Socolov, D. Matorin, and P. Venediktov, "Delayed luminescence yield kinetics in flash illuminated green plants," Plant science letters 19, 339-346 (1980).

10. P. Falkowski and D. A. Kiefer, "Chlorophyll a fluorescence in phytoplankton: relationship to photosynthesis and biomass," J. Plank. Res. 7, 715-731 (1985).
11. Z. Kolber and P. Falkowski, "Use of active fluorescence to estimate phytoplankton photosynthesis in situ," Limnol. Oceanogr. 38, 1646-1665 (1993).

12. P. Falkowski and J. Raven, Aquatic Photosynthesis, (Blackwell Science, 1997).

13. V. Goltsev, P. Chernev, I. Zaharieva, P. Lambrev, and R. J. Strasser, "Kinetics of delayed cholorophyll a fluorescence registered in the ms time range," Photosynth. Res. 84, 209-215 (2005).

14. T. J. Bensky (Department of Physics, California Polytechnic State University, San Luis Obispo, California 93407), L. Chemo, C. Gilbert, B. Neff, M. A. Moline, I. Robbins, and D. Rohan are preparing a paper to be called "Streak camera apparatus for instantaneous observation of nanosecond laser induced fluorescence of in vitro seawater."

15. Crystalaser, 4750 Longley Ln., Suite 205, Reno, Nevada 89502, www.crystalaser.com. Laser model \#QB440.

16. Photek Inc., 26 Castleham Road St, Leonards on Sea, East Sussex, TN38 9NS, United Kingdom, www.photek.com.

17. Stanford Research Systems, 1290-D Reamwood Ave., Sunnyvale, California 94089, www.thinksrs.com, Model \#DG535.

18. See "ImageJ" software at http://rsb.info.nih.gov/ij/.

19. A readily available $685 \mathrm{~nm}$ continuous-wave diode laser was used for this calibration step. Power was controlled via neutral density filters.

20. D. R. James, A. Siemiarczuk, and W. R. Ware, "Stroboscopic optical boxcar technique for the determination of fluorescence lifetimes,” Rev. Sci. Instrum. 63, 1710-1716 (1992).

21. Govindjee, J. Amesz, and D. C. Fork, Light Emission by Plants and Bacteria (Academic, 1986).

22. N. G. Bukhov, U. Heber, C. Wiese, and V. A. Shuvalov, "Energy dissipation in photosynthesis: does the quenching of chlorophyll fluorescence originate from antenna complexes of photosystem II or from the reaction center?," Planta 212, 749-758 (2001).

23. V.Goltsev, I.Zaharieva,P.Lambrev,I. Yordanov, and R. Strasser, "Simultaneous analysis of prompt and delayed chlorophyll a fluorescence in leaves during the induction period of dark to light adaptation,” J. Theor. Biol. 225, 171-183 (2003).

24. I. Zaharieva, V. Goltsev, "Advances on photosystem II investigation by measurement of delayed fluorescence by a phosphoroscopic method," Photochem. Photobiol. 77, 292-298 (2003).

25. J. Haveman and J. Lavorel, "Identification of the 120 microsecond phase in the decay of delayed fluorescence in spinach chloroplasts and subchloroplast particles as the intrinsic back reaction. The dependence of the level of phase on the thylakoids internal pH," Biochim. Biophys. Acta, Mol. Basis Dis. 408, 269-283 1975).

26. K. K. Karukstis and K. Sauer, "Fluorescence decay kinetics of chlorophyll in photosynthetic membranes," J. Cell Biochem. 23, 131-158 (1983).

27. W. A. Arnold, "Experiments," Photosynth. Res. 27, 73-82 (1991).

28. Ocean Optics, 830 Douglas Ave., Dunedin, Florida 34698, USA, www.oceanoptics.com, see the USB-4000 spectrometer

29. L. Valkunas, N. E. Geacintov, L. France, and J. Breton, "The dependence of the shapes of fluorescence induction curves in chloroplasts on the duration of illumination pulses," Biophys. J. 59, 397-408 (1991).

30. A. C. Ley and D. Mauzerall, "Absolute absorption cross sections for photosystem II and the minimum quantum requirement for photosynthesis in Chlorella vulgaris," Biochim. Biohys. Acta 680, 95-106 1982).

31. Z. S. Kolber, O. Prasil, and P. G. Falkowski, "Measurements of variable chlorophyll fluorescence using fast repetition rate techniques: defining methodology and experimental protocols," Biochim. Biophys. Acta Mol. Basis Dis. 1367, 88-106 (1998). 
32. D. Mauzerall, "Light-induced fluorescence changes in chlorella, and the primary photoreactions for the production of oxygen," Proc. Natl. Acad. Sci. USA 69, 1358-1362 (1972).

33. Hydroid, LLC, 6 Benjamin Nye Circle, Pocasset, Massachusetts 02559-4900, www.hydroid.com.

34. U. Uehlinger, "An in situ pulse light fluorimeter for chlorophyll determination as a monitor for vertical and horizontal phyto- plankton distribution in lakes," J. Plank. Res. 7, 605-615 (1985).

35. M. M. Dekshenkis, P. L. Donaghay, J. M. Sullivan, J. E. B. Rines, T. R. Osborn, and M. S. Twardowski, "Temporal and spatial occurrence of thin phytoplankton layers in relation to physical processes," Mar. Ecol. Prog. Ser. 223, 61-71 (2001). 